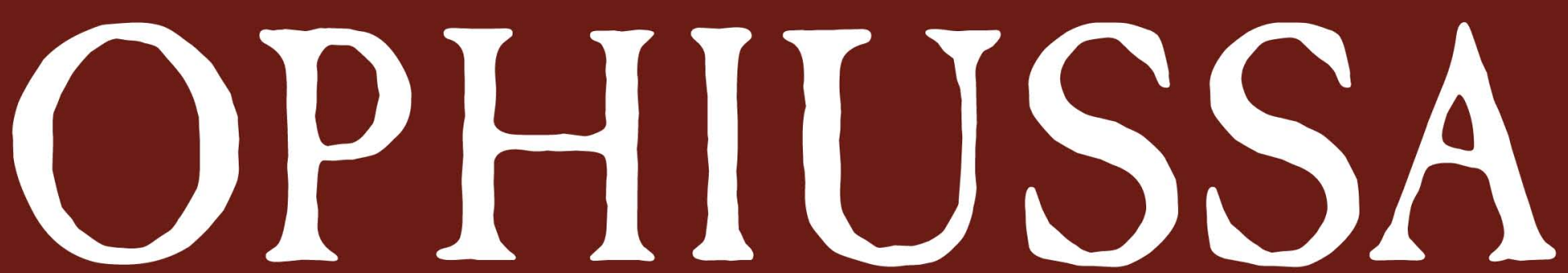

REVISTA DO CENTRO DE ARQUEOLOGIA DA UNIVERSIDADE DE LISBOA

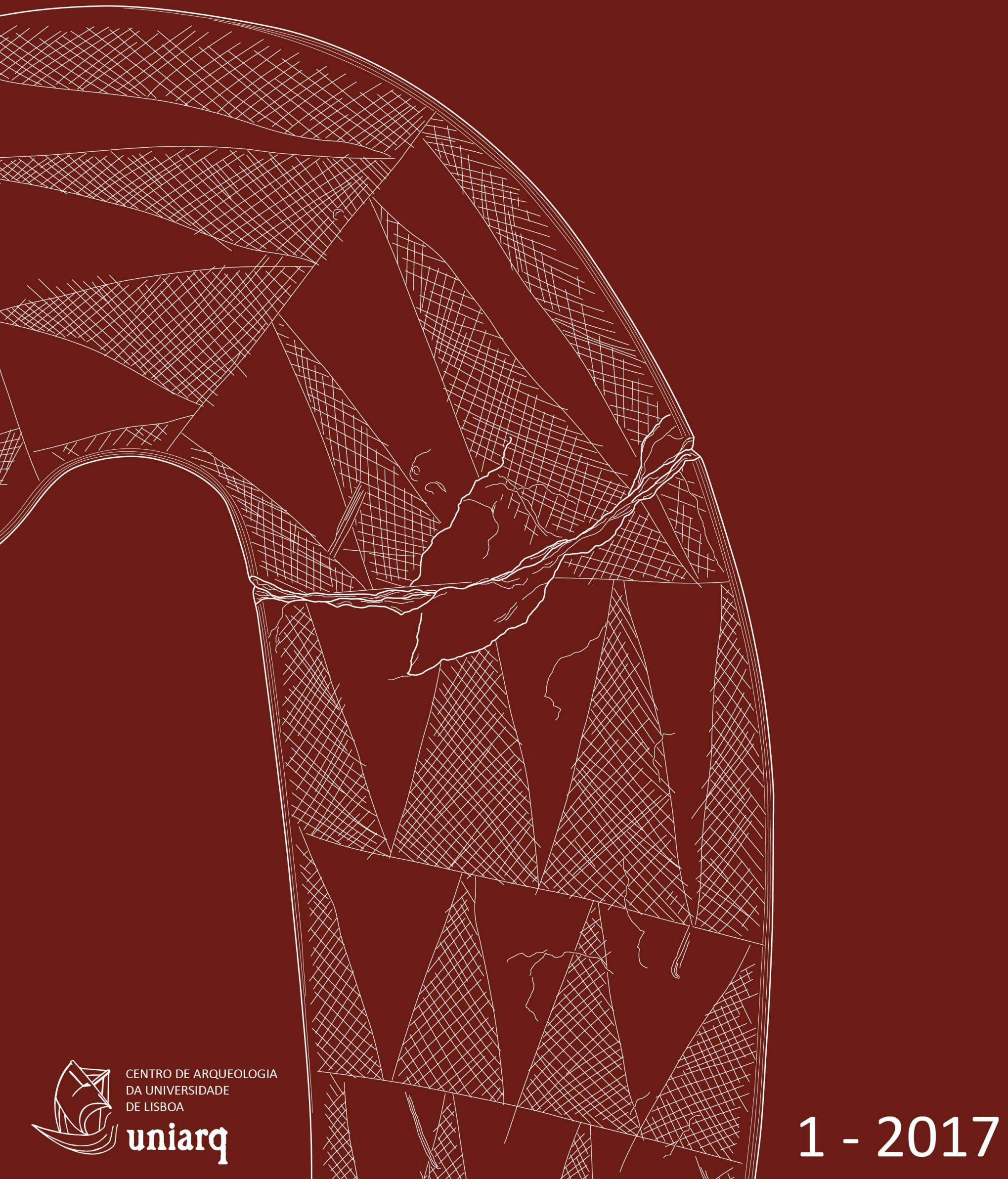


OPHIUSSA. Revista do Centro de Arqueologia da Universidade de Lisboa

ISSN $1645-653 X$

Publicação anual

Volume 1 - 2017

Direcção e Coordenação Editorial:

Ana Catarina Sousa

Elisa Sousa

Rui Boaventura

Conselho Científico:

André Teixeira (Universidade Nova de Lisboa)

Carlos Fabião (Universidade de Lisboa)

Catarina Viegas (Universidade de Lisboa)

Gloria Mora (Universidad Autónoma de Madrid)

Grégor Marchand (Centre National de la Recherche Scientifique)

João Pedro Bernardes (Universidade do Algarve)

José Remesal (Universidade de Barcelona)

Leonor Rocha (Universidade de Évora)

Manuela Martins (Universidade do Minho)

Maria Barroso Gonçalves (Instituto Superior de Ciências do Trabalho e da Empresa)

Mariana Diniz (Universidade de Lisboa)

Raquel Vilaça (Universidade de Coimbra)

Xavier Terradas Battle (Consejo Superior de Investigaciones Científicas)

Secretariado: André Pereira

Capa: André Pereira sobre Báculo do Sobral do Martim Afonso (desenho de Marco Andrade).

Paginação: Elisa Sousa

Impressão: Europress

Data de impressão: Novembro de 2017

Edição impressa (preto e branco)

200 exemplares

Edição digital (a cores)

www.ophiussa.letras.ulisboa.pt

ISSN: $1645-653 X$

Depósito legal: 190404/03

Copyright $\odot 2017$, os autores

Edição:

UNIARQ - Centro de Arqueologia da Universidade de Lisboa

Faculdade de Letras de Lisboa

1600-214 - Lisboa

www.uniarq.net - www.ophiussa.letras.ulisboa.pt - uniarq@letras.ulisboa.pt

O cumprimento do acordo ortográfico de 1990 foi opção de cada autor. 


\title{
OPHIUSSA
}

\section{OPHIUSSA REGRESSA, EM DIGITAL E, LOGO DE SEGUIDA, EM PAPEL}

VICTOR S. GONÇALVES

\author{
Die Vögel hocken still in ihren Zweigen. \\ Die Welt schläft ein! \\ Es wehet külh im Schatten meiner Fichten! \\ Ich stehe hier und harre meines Freundes. \\ Ich harre sein zum letzen Lebewohl.*
}

Gustav Mahler, Das Lied von der Erde (Der Abschied), segundo Hans Bethge, A flauta chinesa. Cantado por Jonas Kaufman, Sony Classical, 2017, 88985389832

Após várias tentativas (sem êxito) para a UNIARQ editar uma Revista própria, após uma colaboração sistemática na CLIO, seguiu-se o número único da CLIO/ARQUEOLOGIA e a OPHIUSSA, número zero, com um nome bem adequado à terra que é a nossa...

Apesar da falta de êxito (e de dinheiro) evidente, desisti de convencer o Rui Boaventura (1971-2016) que às três chega e cedi à sua insistente proposta de disponibilizar online (com uma versão impressa, sobretudo para Arquivo e intercâmbio...) um repositório destinado a reunir aspectos das actividades de investigação do Centro.

Só quem não conheceu o Rui - e alguns dos meus colaboradores mais próximos - poderia pensar que poderia ser de outra maneira. Por alguma razão misteriosa tenho tendência a reunir à minha volta pessoas (extremamente) teimosas e, pior ainda, não Ihes conseguir resistir. O Rui fez comigo a licenciatura, o mestrado e o doutoramento. E nunca consegui convencê-lo em escolher alternativas, apesar de ter sempre tentado.

Trabalhador incansável, com um olhar perspicaz, fascinado por todos os novos contributos em Arqueologia, o Rui, que a Grande Ceifeira tão cedo colheu, merecia ter visto a OPHIUSSA regressar. Não foi possível. Resta-nos reconhecer que continua connosco, em tempos de paz e de guerra, como esteve desde cedo. Esta fotografia, que fiz com a máquina do Miguel Kunst, recorda uma das suas últimas intervenções, neste caso no workshop sobre o casal Leisner em Portugal.

A história das publicações da UNIARQ confunde-se com a sua própria história (e Pré-História...). Em 1974, na sequência da Revolução de Abril, foi extinto o Instituto para a Alta Cultura e, por arrasto, todos os Centros de Investigação que dele dependiam. Na Faculdade de Letras de Lisboa o Centro de Estudos Históricos também encerrou. De portas abertas, foi saqueado e escapou por pouco às partilhas de território que a Faculdade então presenciou. Com Joaquim Barradas de Carvalho e António Borges Coelho, imaginámos um novo Centro, onde a História e a Arqueologia tivessem lugar (e a História da Arte também). Mas hienas e sardinhas não gostaram e o Centro começou agitado, tal como os tempos que se viviam numa Universidade que

* «Os pássaros abrigam-se, silenciosos, na ramagem/ O mundo adormece/ Uma brisa fresca passa na sombra dos meus pinheiros/ Estou aqui, espero o meu amigo/ Espero-o para um último adeus.». 


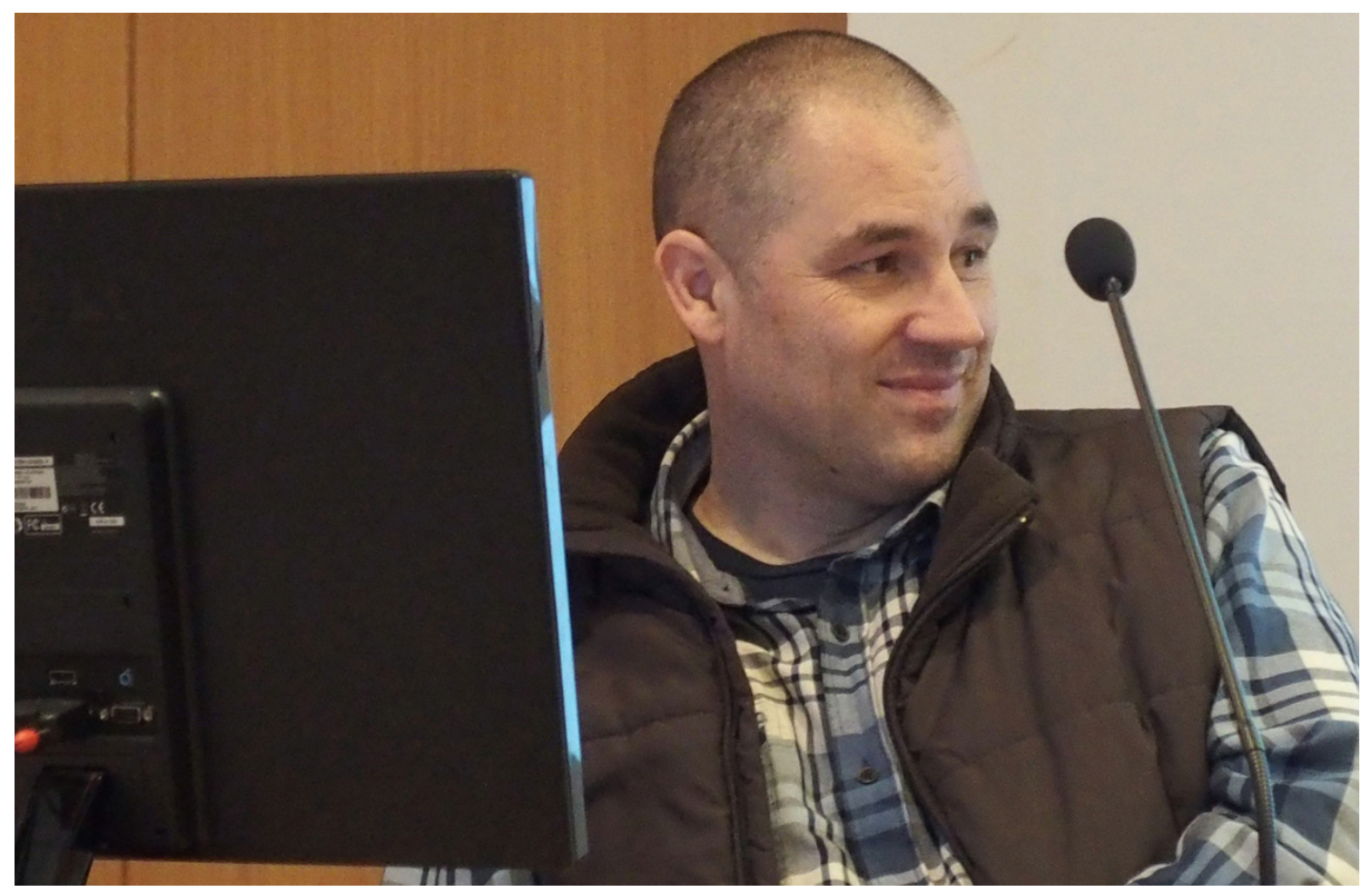

Rui Boaventura (1971-2016), falando na Faculdade de Letras de Lisboa sobre temas do $3^{\circ}$ milénio a.n.e., com o $4^{\circ}$ milénio os seus períodos pré-históricos preferidos. E onde o casal Leisner teve um decisivo impacto. Foto VSG.

não se conseguia renovar em bloco e onde as tímidas tentativas de sensatez eram tomadas por cobardia e a criatividade era considerada inimiga e perigosa.

No novo Centro de História, que ambiciosamente se chamava inicialmente «das Universidades de Lisboa», aprendeuse a primeira lição, quando, por ordem nem sequer justificada (ainda que óbvia), do Instituto Nacional de Investigação Científica, o plural foi substituído pelo singular e o Centro de História da Universidade de Lisboa iniciou o seu atribulado caminho. A competição entre Faculdades anunciava-se já e ainda não tinha chegado o tempo de Vítor Jabouille, António Feijó e Paulo Alberto, por natureza ou convicção, abertos à transformação e mudança, ainda que de formas distintas.

E nesse novo Centro, de cuja direç̧ão participei até à Grande Secessão, a questão das publicações foi-me entregue.

Os quatro primeiros números da Revista que criei, a CLIO, apresentavam já propostas gráficas inovadoras e, não havendo então muitos designers em Portugal, um País onde tardaram demasiado a aparecer, passei horas e horas a montar páginas, a recortar linguados (mas com uma tesoura e não com uma faca de filetear da Global ou da Wüsthof, como hoje faço...), colando as colunas à medida do previsto, em folhas A4.

Os volumes da CLIO «mista» tiveram os seguintes índices:

\section{CLIO - REVISTA DO CENTRO DE HISTORIA DA UNIVERSIDADE DE LISBOA - VOL. 1 - 1979}

ÍNDICE

Por uma nova História do pensamento . .7

Joaquim Barradas de Carvalho

Une Voie de Connaissance pour l'histoire de la société portugaise au XVIIle Siècle: Les Micro-Biographies (sourcesméthode-étude de cas) 21

Andrée Mansuy-Diniz Silva

Questões de administração local nas Inquirições Gerais de Afonso IV. . .69

Maria José Lagos Trindade
O Cardeal-Rei numa fonte coeva . .81

Francisco Salles Loureiro

Notas para a história dos partidos políticos em Portugal no período do «Ministério Da Regeneração» (1851-1856).....91 António Ravara

A Carta Arqueológica do Algarve.

Victor dos Santos Gonçalves

As Ânforas de S. Bartolomeu de Castro Marim. .141

Manuel Maia

Notas de Introdução a uma leitura de Sérgio 155 
Perspectivas actuais da historiografia medieval espanhola-

J. A. Garcia De Cortázar. . .161

José Mattoso

O Ano do Património Arquitectónico Europeu. . .169

Jorge H. Pais Da Silva

\section{NOTICIÁRIO}

Conferências, Colóquios e Seminários Promovidos Pelo Centro de História.

Importantes descobertas arqueológicas no povoado da idade do cobre de Vidais (Marvão). 178

Escavações em povoados da idade do cobre no Alto Algarve

Oriental. 179

Roteiro de fontes de História Contemporânea de Portugal .179

\section{VÁRIA}

Centro de História da Universidade de Lisboa. .183

In Memoriam . .185

Pedro Barbosa

IV Congresso Nacional De Arqueologia.

Projecto de acordo entre o Centro de História da Universidade de Lisboa e o Museu de Arqueologia e Etnografia do Distrito de Setúbal. ..189

Projet de convention entre le Centre d'anthropologie des Sociétés Rurales de L'École des Hautes Etudes en Sciences Sociales et le Centre d'Histoire.

\section{CLIO - REVISTA DO CENTRO DE HISTORIA DA UNIVERSIDADE DE LISBOA - VOL. 2 - 1980}

\section{ÍNDICE}

\section{ABERTURA}

Notas (para um artigo que não chegou a ser feito)................7 Joaquim Barradas de Carvalho

\section{ESTUDOS \& INTERVENÇÕES}

Espaço e Tempo. Evolução do ambiente geográfico de Portugal ao longo dos tempos pré-históricos . .13

Suzanne Daveau

O Dólmen de S. Pedro Dias (Poiares). .39

A. Augusto Tavares

A Necrópole da Idade do Bronze do Monte de Vale de Carvalho (Sítimos).

Ana Margarida Arruda, Victor Gonçalves, F. Bragança Gil e Gaspar Ferreira

Povos do Sul de Portugal nas Fontes Clássicas - Celtici e Turduli.

Manuel Maia

O Sítio Romano-Árabe do Vale do Boto. Notícia da Sua Identificação... . .71 Victor Gonçalves, Helena Catarino e Ana Margarida Arruda La Presse de La Democratie Chrétienne Au Portugal de 1870 à 1913 .. .81

Marie Christine Volovitch

Manuel Teixeira Gomes e Sidónio Pais 117

João Medina

\section{RELATÓRIOS DE ACTIVIDADE}

Cerro do Castelo de Corte João Marques. Escavações de 1979. Relatório sumário dos trabalhos de campo. .131
Victor Gonçalves

Cerro do Castelo de Santa Justa (Alcoutim). Escavações de 1979. Extractos do caderno de campo. . .133 Victor Gonçalves

\section{VÁRIA}

Joaquim Barradas de Carvalho: Para a História de um Historiador. .141

Victor Gonçalves

Em Memória de Joaquim Barradas de Carvalho, sem falar no Historiador..

José-Augusto França

Maria José Trindade .149

\section{NOTICIÁRIO}

Centro de História: resumo de actividades até 1980.......153

O IV Congresso Nacional de Arqueologia.

Ana Margarida Arruda

Roteiro das fontes manuscritas da história contemporânea (séculos XVIII-XX) 157

Miriam Halpern Pereira e M. José Silva Leal

Comissão Directiva do Centro de História. Linhas de Acção/ 81

Projecto de acordo entre o Centro de História da Universidade de Lisboa e o Centro de Física Nuclear da Universidade de Lisboa. 158

Escavações na Estação Romana de S. Marcos, Cacém (Sintra). 158 Manuel Maia

Clio 3. 159 

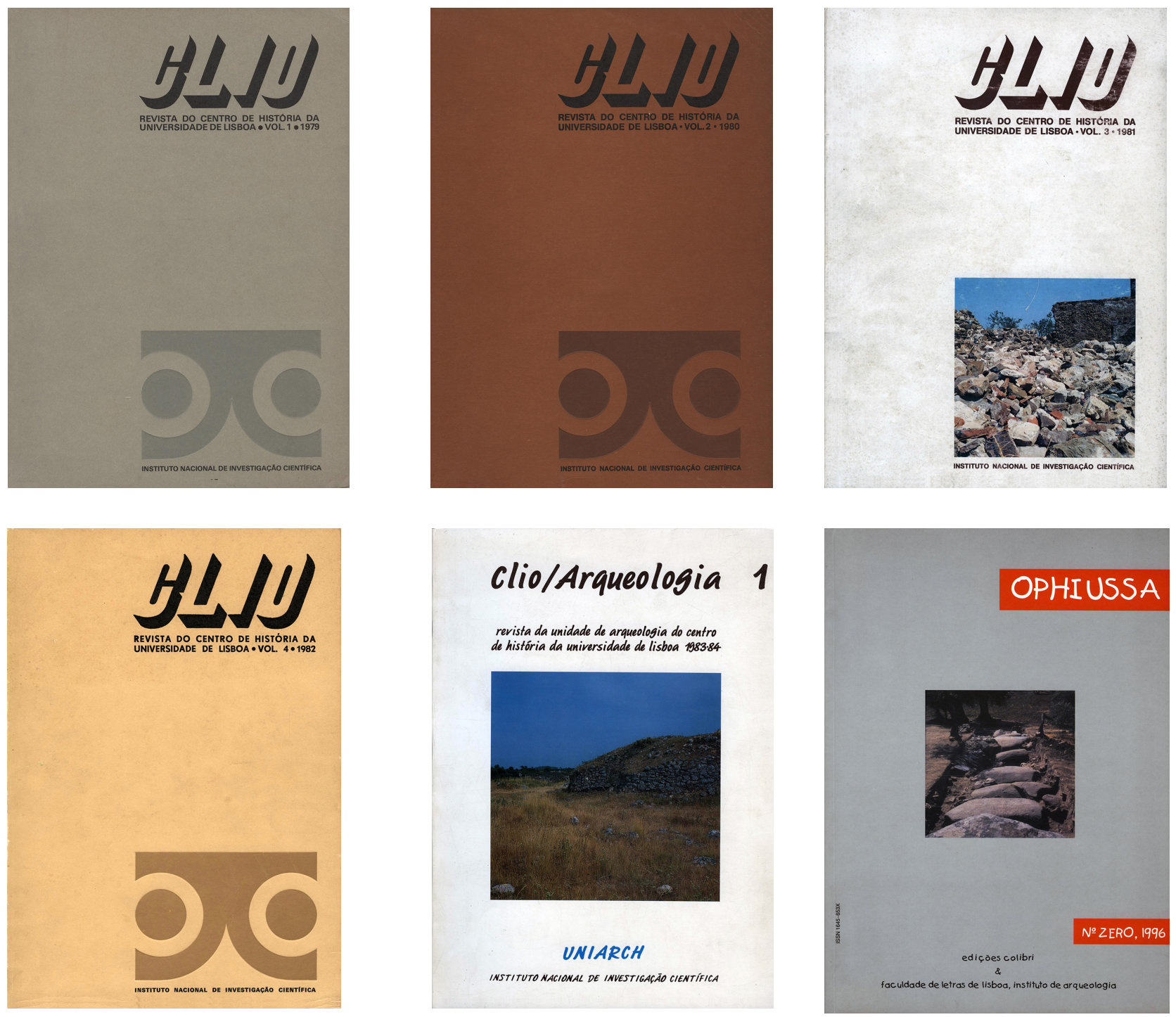

CLIO - REVISTA DO CENTRO DE HISTÓRIA DA UNIVERSIDADE DE LISBOA - VOL. 3 - 1981

ÍNDICE

\section{ABERTURA} .7

\section{ESTUDOS \& INTERVENÇÕES}

Vale do Boto: Escavações de 1981 no complexo árabe/ medieval.....

Helena Catarino, Ana Margarida Arruda e Victor Gonçalves Os Testamentos Régios (Séculos XII A XV), Aspectos Vários... .. .29

M. Fernanda Maurício

O Processo de António Fernandes, Piloto da Guiné, na Inquisição de Lisboa. . .37 Isaías da Rosa Pereira O Interesse por Samatra (...). .49 Luís de Albuquerque Tratado do cerco de Mazagão e do que nele (se) passou..53 Manuela Mendonça

O Problema do casamento do Cardeal-Rei. .67
Francisco Salles Loureiro

A llha da Madeira Setecentista . .83

Maria De Lourdes de Freitas Ferraz

O Zé Povinho Durante A República. .103 João Medina

Acerca das Eleições de 1911

António Pinto Ravara

\section{RELATÓRIOS DE ACTIVIDADE}

Anta dos Penedos de S. Miguel (Crato), Campanha 1 (81)....

Victor Gonçalves, Françoise Treinen-Claustre e Ana Margarida Arruda

Cerro do Castelo de Santa Justa (Alcoutim). Campanha 2 (80).

Victor Gonçalves

Cerro do Castelo de Santa Justa (Alcoutim). Campanha 3 (81)... 


\section{VÁRIA}

Arqueologia do Algarve: Sinopse retrospectiva e perspectivas de mudança. . .177

Victor Gonçalves

Nota acerca de alguns materiais da II Idade do Ferro do

Complexo Arqueológico dos Vidais (Marvão). . .183

Ana Margarida Arruda e Helena Catarino

A Vigia (Medieval) de Paio Peres Correia. . .189

Victor Gonçalves

Um Soldado Português Fuzilado na Flandres. . .193

Aniceto Afonso e Marília Guerreiro

Lembrança de Barradas de Carvalho.

Luís de Albuquerque

\section{LIVROS NOVOS, LEITURAS NOVAS?}

A Dinâmica da Hominização. 205

Victor Gonçalves

As Origens recuperadas?

Victor Gonçalves

A Cerâmica Medieval no Mundo Mediterrânico.

Helena Catarino e Ana Margarida Arruda

Para uma Nova História Urbana.

A. A. Marques De Almeida

NOTICIÁRIO. .215

\section{CLIO - REVISTA DO CENTRO DE HISTORIA DA UNIVERSIDADE DE LISBOA - VOL. 4-1982}

Direcção de Salles Loureiro, João Medina, Victor Gonçalves

\section{ÍNDICE}

\section{ESTUDOS \& INTERVENÇÕES}

O povoado calcolítico do Cabeço do Pé da Erra (Coruche)...7 Victor S. Gonçalves

Materiais campaniformes do concelho de Oliveira do Hospital. . .19

João Carlos de Senna Martinez

Cerâmicas da Idade do Ferro da Alcáçova de Santarém...35

Ana Margarida Arruda

Uma introdução ao Doomesday Book

Fernanda Maurício

Política norte-africana: rumos na expansão portuguesa...51

Maria Claro Junqueiro

Uma carta inédita de Afonso de Albuquerque.

Maria Clara Junqueiro, António Ribeiro Guerra

Jesuítas na crista da onda da política seiscentista . .71

Francisco Salles Loureiro

O crescimento de Lisboa e Porto na segunda metade do

século XIX e princípios de XX.

António Ravara

Arquivos históricos de Lisboa: contribuição para um roteiro. . .95

Arnaldo António Pereira

\section{ENTREVISTA}

Entrevista com Aurélio Quintanilha. 121

João Medina

\section{VÁRIA}

Datation au C14 du site archaeologique de la plage de Magoito. . .133

Suzanne Daveau, Ana Ramos Pereira, Georges Zbyszewski Povoado pré-histórico do Cabeço do Cubo (Campo Mai or).

Jorge Oliveira, Ana Carvalho Dias

Epitáfio de Euprepres.

José d'Encarnação

Um mercúrio em bronze, inédito.

Pedro Barbosa

A propósito de Morón

António Dias Diogo

\section{RELATÓRIOS DE ACTIVIDADE}

Cerro do Castelo de Santa Justa (Campanha 4 (82)...........155 Victor Gonçalves

Escavações arqueológicas na llha do Pessegueiro (3a campanha).................................................................................165

Carlos Tavares da Silva, Joaquina Soares, Antónia Coelho Soares

Após os quatro números da CLIO, veio o único da CLIO/ARQUEOLOGIA, com uma abertura contundente e uma organização bem diferente. Curioso é notar que o cartoon de Fred que abria a revista foi mal entendido pelo ego de um arqueólogo de então, que supunha ver-se nele representado, dado o ambiente marinho.

Na verdade, era o director da Revista que queria mostrar a utilidade de ser puxado, ao mesmo tempo, de direcções muito diferentes. O equilíbrio de essas forças garantia que ele continuasse tranquilo, no lugar e caminho que para si escolheu... 

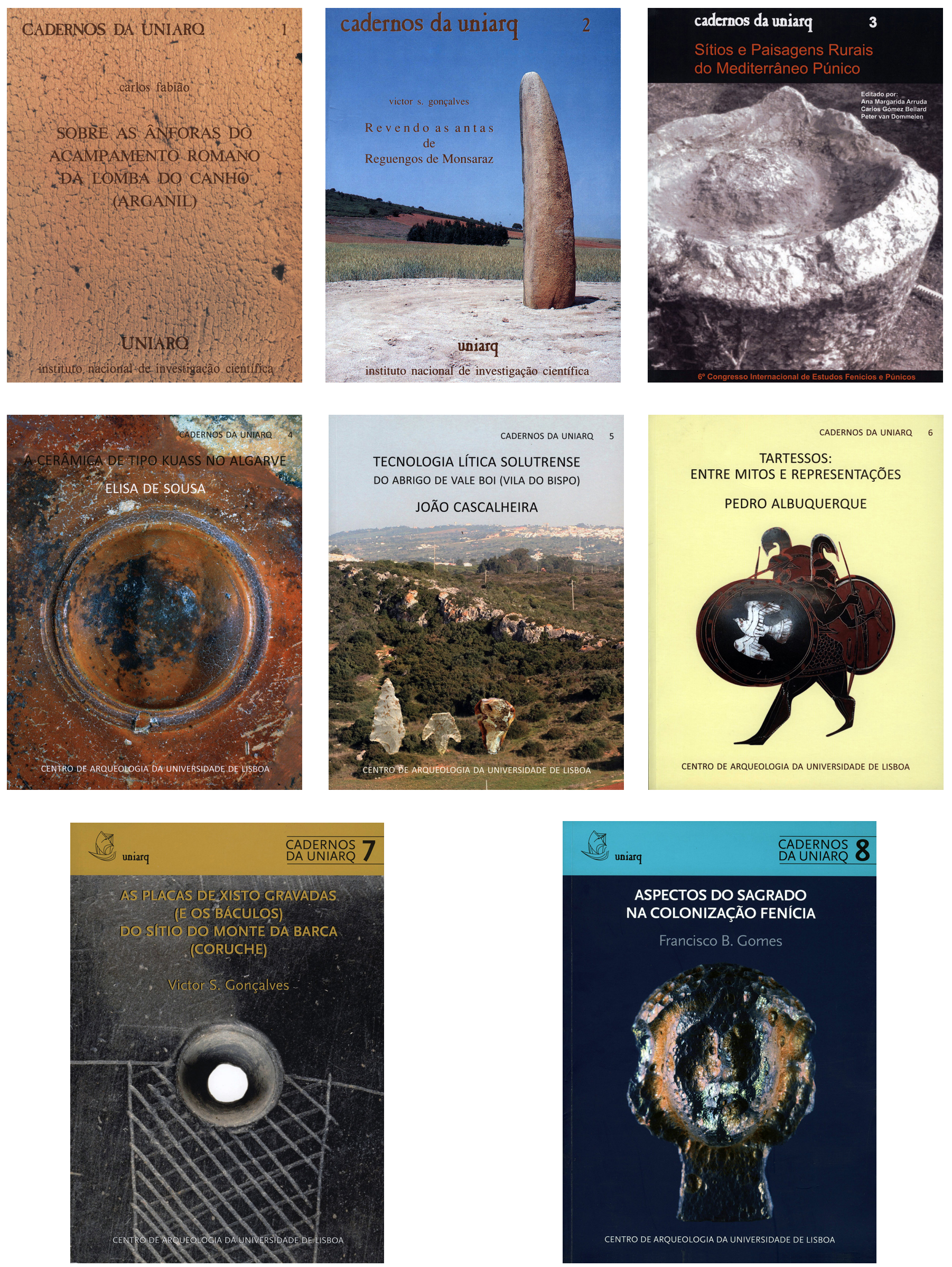


\section{ÍNDICE DE CLIO/ARQUEOLOGIA 1}

\section{EDITORIAL}

APRESENTAÇÃO, SEGUIDA DE UMA PAVANA POR UMA ARQUEOLOGIA (QUASE) DEFUNTA, COM VOTOS DE PRONTO RESTABELECIMENTO. VICTOR GONÇALVES

\section{ESTUDOS E INTERVENÇÕES}

UM CORTE ATRAVÉS DA FORTIFICAÇÃO INTERIOR DO CASTRO CALCOLÍTICO DE VILA NOVA DE S. PEDRO, SANTARÉM (1959)... 19-29 H. N. SAVORY

A CRONOLOGIA ABSOLUTA (DATAÇÕES C14) DE ZAMBUJAL $31-40$

H. SCHUBART E E. SANGMEISTER

O POVOADO CALCOLÍTICO DE LECEIA (OEIRAS), 1. a E 2. ${ }^{a}$ CAMPANHAS DE ESCAVAÇÃO $(1982,1983)$ 41-68 JOÃO L. CARDOSO, JOAQUINA SOARES E CARLOS TAVARES DA SILVA

CABEÇO DO PÉ DA ERRA (CORUCHE), CONTRIBUIÇÃO DA CAMPANHA 1(83) PARA O CONHECIMENTO DO SEU POVOAMENTOCALCOLÍTICO. .69-75 VICTOR GONÇALVES

RESUMOS DE INTERVENÇÕES EM ESCOURAL (MONTEMOR-O-NOVO) E MONTE DA TUMBA (TORRÃO) $.77-79$

ROSA E MÁRIO VARELA GOMES, M. FARINHA DOS SANTOS, JOAQUINA SOARES E CARLOS TAVARES DA SILVA

DOZE DATAS 14C PARA O POVOAMENTO CALCOLÍTICO DO CERRO DO CASTELO DE SANTA JUSTA (ALCOUTIM): COMENTÁRIOS E CONTEXTOS ESPECÍFICOS. $.81-92$ VICTOR GONÇALVES

PRECISIONES EN TORNO DE LA CRONOLOGIA ANTIGUA DE PAPA UVAS (ALJARAQUE, HUELVA). ..93-104

J. C. MARTÍN DE LA CRUZ

CONTRIBUIÇÕES PARA UMA TIPOLOGIA DA OLARIA DO MEGALITISMO DAS BEIRAS: OLARIA DA IDADE DO BRON ZE. 105-138

J. C. SENNA-MARTÍNEZ

\section{EM DISCUSSÃO}

POVOADOS CALCOLÍTICOS FORTIFICADOS NO CENTRO/SUL DE PORTUGAL: GÉNESE E DINÂMICA EVOLUTIVA. 141-154 VICTOR GONÇALVES, JOÃO CARDOSO, ROSA EMÁRIO VARELA GOMES, ANA MARGARIDA ARRUDA, JOAQUINA SOARES, CARLOS TAVARES DA SILVA, CAETANO DE MELLO BEIRÃO, RUI PARREIRA

ARQUEOLOGIA HOJE (CONVERSAS DE ARQUEOLOGIA \& ARQUEÓLOGOS)

JEAN GUILAINE RESPONDE A VICTOR GONÇALVES....157-166

\section{MEDIR E CONTAR}

CONTRIBUIÇÕES ARQUEOMÉTRICAS PARA UM MODELO SOCIO-CULTURAL:PADRÕESVOLUMÉTRICOS NA IDADEDO
BRONZE DO CENTRO E NW DE PORTUGAL $169-188$ J. C. SENNA-MARTÍNEZ

\section{VARIA ARCHAEOLOGICA}

TRÊS INTERVENÇÕES SOBRE ARQUEOLOGIA NO ALGARVE. 191-196 VICTOR GONÇALVES, ANA MARGARIDA ARRUDA, HELENA CATARINO

ARTEFACTO DE PEDRA POLIDA DE GRANDES DIMENSÕES PROVENIENTE DE ALMODÔVAR (BEJA). 197-199 VICTOR GONÇALVES

\section{EM CONSTRUÇÃO. RELATÓRIOS DE ACTIVIDADE}

PROGRAMA PARA O ESTUDO DA ANTROPIZAÇÃO DO BAIXO TEJO E AFLUENTES: PROJECTO PARA O ESTUDO DA ANTROPIZAÇÃO DO VALE DO SORRAIA (ANSOR)....203-206 VICTOR GONÇALVES, SUZANNE DAVEAU

PROGRAMA PARA O ESTUDO DA EVOLUÇÃO DAS SOCIEDADES AGRO-PASTORIS, DAS ORIGENS À METALURGIA PLENA, DOS ESPAÇOS ABERTOS AOS POVOADOS FORTIFICADOS, NO CENTRO DE PORTUGAL (ESAG). 207-211 VICTOR GONÇALVES

O MONUMENTO N. 3 DA NECRÓPOLE DOS MOINHOS DE VENTO, ARGANIL - A CAMPANHA 1(84). 213-216

J. C. SENNA-MARTÍNEZ

ALCÁÇOVA DE SANTARÉM. RELATÓRIO DOS TRABALHOS ARQUEOLÓGICOS DE 1984 217-223

ANA MARGARIDA ARRUDA

ANTA DOS PENEDOS DE S. MIGUEL (CRATO). CAMPANHA 2(82). $.225-227$

VICTOR GONÇALVES, FRANÇOISE TREINEN-CLAUSTRE, ANA MARGARIDA ARRUDA, JEAN ZAMMIT

ANTA DOS PENEDOS DE S. MIGUEL (CRATO). CAMPANHA 3(83). 229-230 VICTOR GONÇALVES, FRANÇOISE TREINEN-CLAUSTRE, ANA MARGARIDA ARRUDA, JEAN ZAMMIT

CERRO DO CASTELO DE SANTA JUSTA (ALCOUTIM). CAMPANHA 5(83). OBJECTIVOS, RESULTADOS, PERSPECTIVAS.............................................................231-236

VICTOR GONÇALVES

CERRO DO CASTELO DE SANTA JUSTA (ALCOUTIM). CAMPANHA 6(84). RESUMO DE CONCLUSÕES...........237-243 VICTOR GONÇALVES

ESCAVAÇÕES ARQUEOLÓGICAS NO CASTELO DE CASTRO MARIM. RELATÓRIO DOS TRABALHOS DE 1983........245-248 ANA MARGARIDA ARRUDA

ESCAVAÇÕES ARQUEOLÓGICAS NO CASTELO DE CASTRO MARIM. RELATÓRIO DOS TRABALHOS DE 1984.........249-254 ANA MARGARIDA ARRUDA

\section{LIVROS NOVOS, NOVOS LIVROS}

PARA UMA ARQUEOLOGIA TOTAL

LUÍS GONÇALVES, PAULA FERREIRINHA $.257-259$ 


\section{ANTAS DO CONCELHO \\ $D E$}

REGUENGOS DE MONSARAZ

GEORG e VERA LEISNER

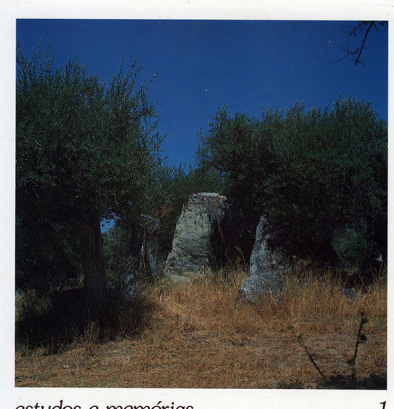

UNIARCH

instituto nacional de investigação científica
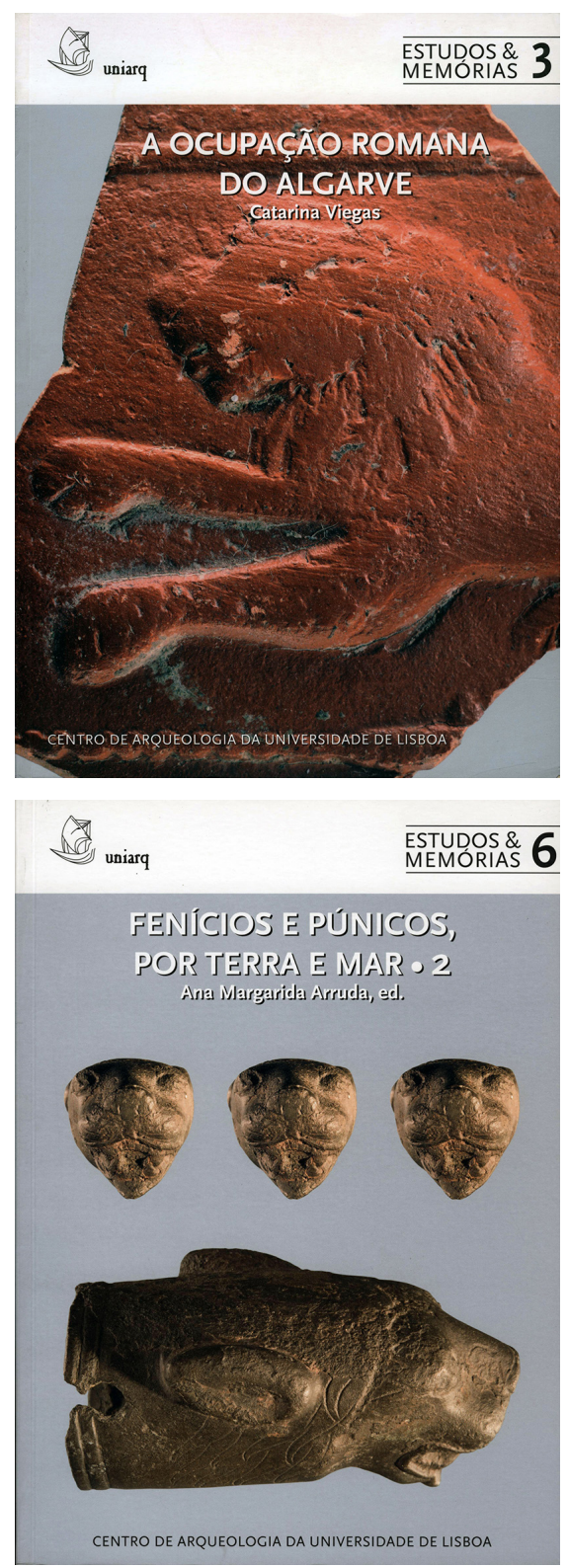

MEGALITISMO E METALURGIA MO ALTO ALGARVE ORIEMTAL

uma aproximaçăo integrada

Victor S. Gonçalves

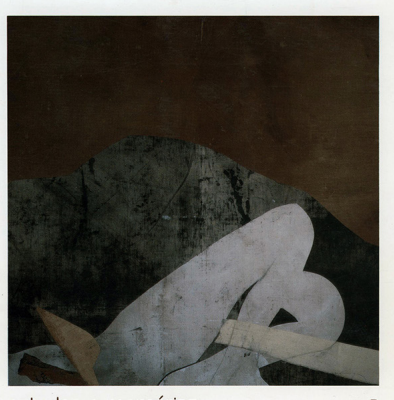

estudos e memórias

instituto nacional de investigação científica
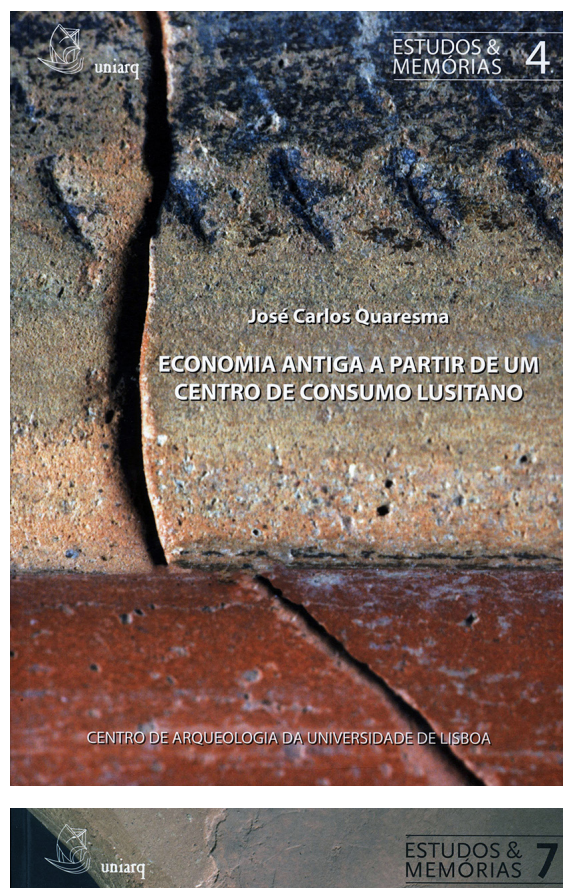

A OCUPACÃO PRÉ-ROMANA DA FOZ DO ÉSTUÁRIO DO TEJO E. Elisa de Sousa

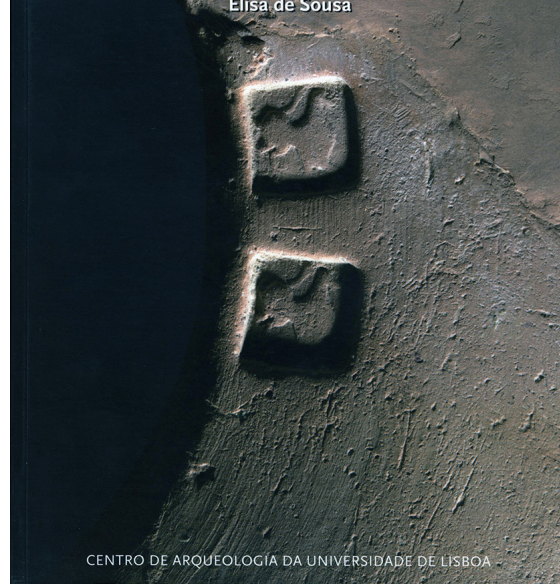

MEGALITISMO E METALURGIA MO ALTO ALGARVE ORIENTAL uma aproximação integrada

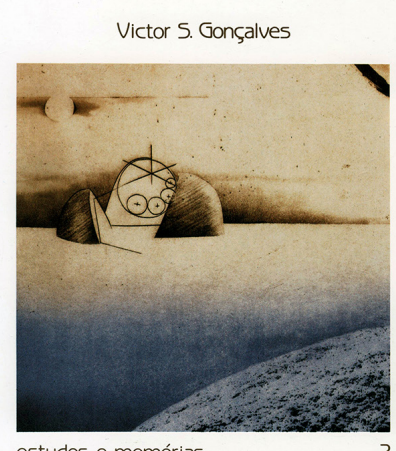

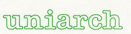

instituto nacional de investigação científica
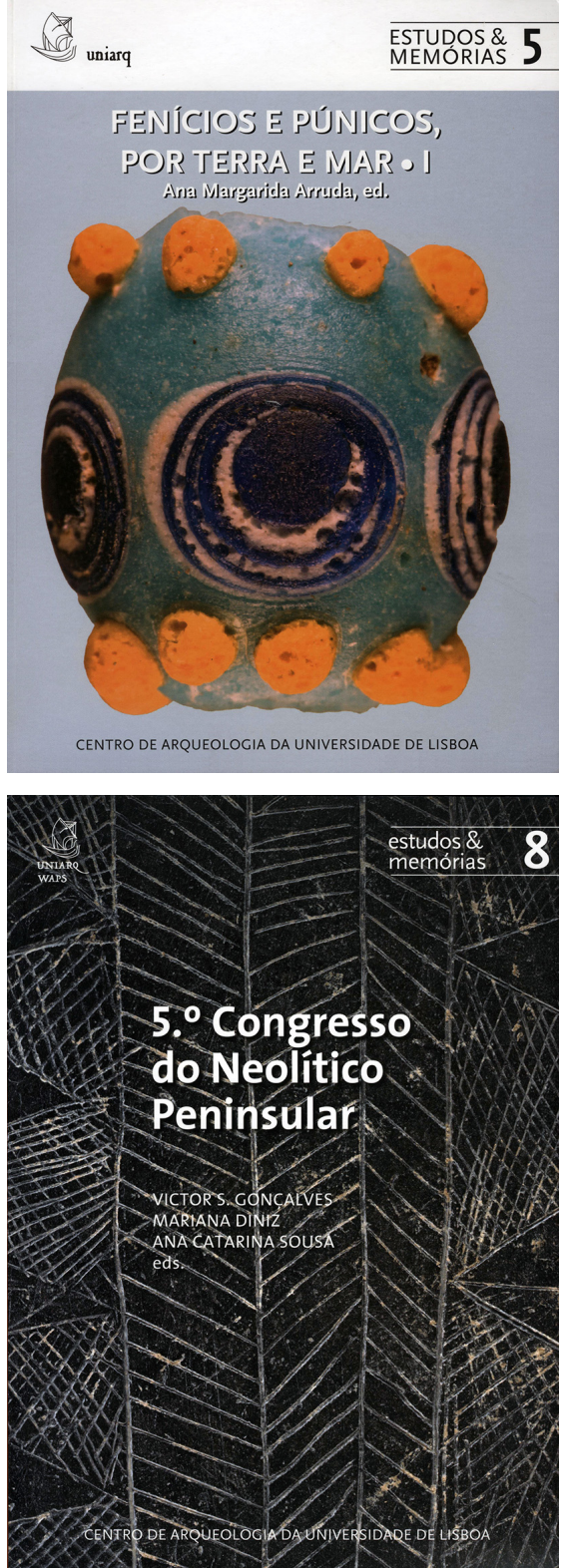


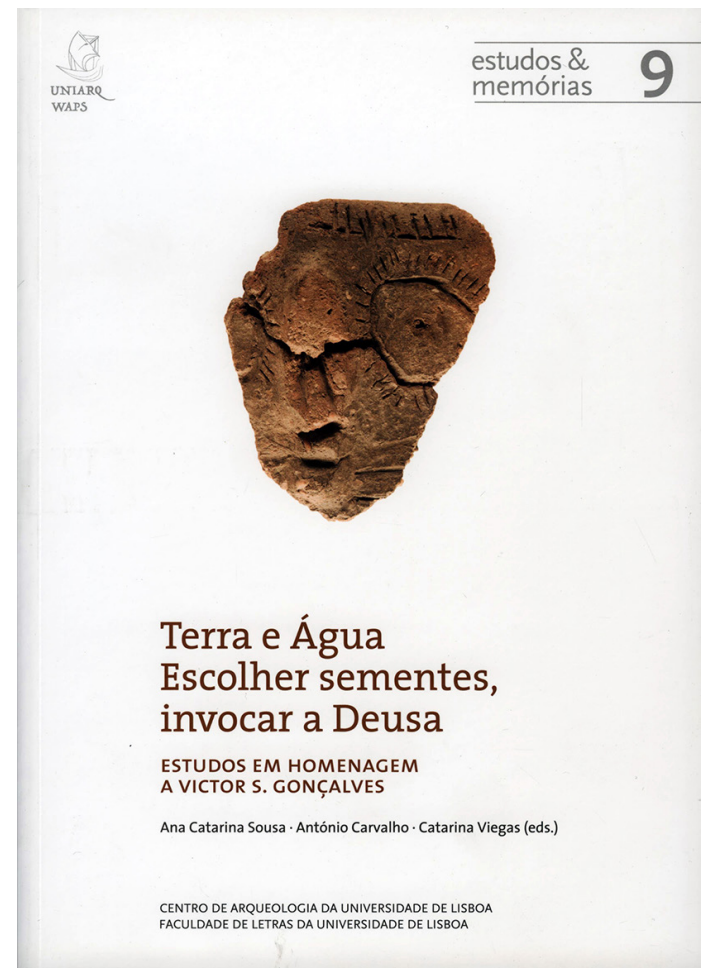

PRÉ-HISTÓRIA E DECADÊNCIA

DOUTORAMENTO EM PRÉ-HISTÓRIA

$.274-276$

TERESA GOMES DA COSTA, ANTÓNIO BAPTISTA NOVO DOUTORAMENTO EM ARQUEOLOGIA

PRÉ-HISTÓRIA EUROPEIA, ENTRE O ENSAIO E O MITO.

NUNO CARVALHOSANTOS

VILA NOVA DE S. PEDRO: O RECOMEÇO.

PUBLICAÇÕES DA UNIARCH

GOVERNADOR CIVIL DE FARO VISITA ESCAVAÇÕES DO CERRO DO CASTELO DE SANTA JUSTA

\section{NOTÍCIAS E RECORTES}

AS PRIMEIRAS COMUNIDADES RURAIS NO MEDITERRÂNEO OCIDENTAL 267-269

COMISSÃO DIRECTIVA DO CENTRO DE HISTÓRIA. .269

QUINTA DO LAGO, UMA INTERVENÇÃO DE EMERGÊNCIA

DA UNIARCH.

270-271

A UNIARCH E O PROJECTO ANSOR EM CORUCHE....272-273

\section{EM ANEXO}

TEXTOS DE ARQUEOLOGIA EM CLIO, REVISTA DO CENTRO DE HISTÓRIA DA UNIVERSIDADE DE LISBOA (1979-1982).

Anos depois do número único da CLIO/ARQUEOLOGIA, veio OPHIUSSA ZERO, com outra Abertura áspera, ela própria dedicada a Vila Nova de S. Pedro.

O seu Índice era assim:

VICTOR S. GONÇALVES, algumas histórias exemplares (e outras menos)

MARIANA DINIZ, A Arqueologia Pós-Processual ou o passado pós-moderno

JOÃO ZILHÃO e Francisco Almeida, Interpretação Tecnológica e paletnográfica da ocupação protosolutrense da Lapa do Anecrial (Porto de Mós) JOAQUINA SOARES, Para uma reconstrução do processo de neolitização em Portugal

ANA CATARINA SOUSA, O Megalitismo da Galiza. Notas para uma bibliografia crítica
JOÃO CARLOS DE SENNA-MARTíNEZ, Do espaço doméstico ao espaço funerário: ideologia e cultura material na préhistória recente do centro de Portugal

VICTOR S. GONÇALVES, Pastores, agricultores e metalurgistas em Reguengos de Monsaraz: os $4^{\circ}$ e $3^{\circ}$ milénios

MANUEL CALADO, Endovélico e Rocha da Mina - o contexto arqueológico

Carlos Fabião, Amílcar Guerra, A Cerâmica Campaniense do Acampamento Romano da Lomba do Canho (Arganil) AMÍLCAR GUERRA; CARLOS FABIÃO, A ocupação romana 
do Cabeço do Crasto, S. Romão, Seia

VICTOR S. GONÇALVES; ANA MARGARIDA ARRUDA; MANUEL CALADO, Novos contributos para a arqueologia do Algarve oriental
CARLOS TAVARES DA SILVA, Os sítios, «horizontes»e artefactos de Victor S. Gonçalves

Estamos cá todos, ainda e por enquanto, com alguns mais a chegarem e outros a partirem.

Olhando para o índice do número 1 de OPHIUSSA, que ora se apresenta, teremos,

MARCO ANTÓNIO ANDRADE - O sítio pré-histórico do Sobral do Martim Afonso (Salvaterra de Magos, Portugal): um curioso contexto do Neolítico Final/ Calcolítico na margem esquerda do Baixo Tejo;

ANA CATARINA SOUSA e JORGE LOPES - O sítio do Moinho do Custódio (Arruda dos Vinhos): leituras preliminares e algumas considerações sobre o povoamento calcolítico na Península de Lisboa;

PEDRO ALBUQUERQUE - O Guadiana como fronteira? Notas para um projecto de investigação;

ANA MARGARIDA ARRUDA, ELISA DE SOUSA, JOÃO PIMENTA, RUI SOARES, HENRIQUE MENDES - Fenícios e indígenas em contacto no Estuário do Tejo;
ELISA DE SOUSA - Algumas reflexões sobre a fase tardia da Idade do Ferro no Ocidente Atlântico;

CARLOS PEREIRA - Produção e comércio de lucernas durante a Antiguidade Tardia: génese e evolução das lucernas tardo-antigas de produção Africana;

JACINTA BUGALHÃO - O papel da mulher na Arqueologia Portuguesa;

RUI BOAVENTURA - VERA.LEISNER@PORTUGAL.PT;

e, na secção de RECENSÕES BIBLIOGRÁFICAS, - Estudos Arqueológicos de Oeiras, 22 volumes e a contar... (VICTOR S. GONÇALVES).

Quanto às publicações de outra tipologia, temos as séries Cadernos da uniarq e estudos\&memórias.

Nos primeiros, editaram-se

\section{CADERNOS DA UNIARQ}

1 - FABIÃO, C. (1989) - Sobre as ânforas do acampamento romano da Lomba do Canho (Arganil). ISBN: 972-667-108-6

2 - GONCALVES, V. S. (1992) - Revendo as antas de Reguengos de Monsaraz. ISBN: 972-667-319-4

3 - ARRUDA, A. M.; GOMEZ BELLARD, C.; VAN DOMMELEN, P., eds. (2007) - Sítios e paisagens rurais do Mediterrâneo púnico. ISBN: 978-989-95653-0-2

4 - SOUSA, E. (2009) - A cerâmica de tipo Kuass no Algarve. ISBN: 978-989-95653-1-9

5 - CASCALHEIRA, J. (2010) - Tecnologia lítica solutrense do Abrigo Vale Boi. ISBN: 978-989-95653-3-3

6 - ALBUQUERQUE, P. (2010) - Tartessos: entre mitos e representações. ISBN: 978-989-95653-2-6

7 - GONÇALVES, V. S. (2011) - As placas de xisto gravadas (e os báculos) do sítio do Monte da Barca (Coruche). ISBN: 978-989-95653-5-7

8 - GOMES, F. B. (2012) - Aspectos do sagrado na colonização fenícia. Contextos de culto de influência oriental na Idade do Ferro do Sul de Portugal (séculos VIII-III a.n.e.). ISBN: 978-989-95653-6-4

Dos segundos, publicaram-se

\section{ESTUDOS \& MEMÓRIAS}

1 - LEISNER, G. e LEISNER, V. (1985) - Antas do Concelho de Reguengos de Monsaraz. Estudos \& memórias, 1. Lisboa: Uniarch/ INIC. 321 p. ISBN:

2 - GONÇALVES, V. S. (1989) - Megalitismo e Metalurgia no Alto Algarve Oriental. Uma aproximação integrada. 2 Volumes. Estudos \& memórias, 2. Lisboa: CAH/Uniarch/ INIC. 566+333 p. ISBN: 972-667-109-4

3 - VIEGAS, C. (2011) - A ocupação romana do Algarve. Estudo do povoamento e economia do Algarve central e oriental no período romano. Estudos \& memórias 3. Lisboa: UNIARQ. 670 p. ISBN: 972-989-95653-4-0

4 - QUARESMA, J. C. (2012) - Economia antiga a partir de um centro de consumo lusitano. Terra sigillata e cerâmica africana de cozinha em Chãos Salgados (Miróbriga?). Estudos \& memórias 4. Lisboa: UNIARQ. 488 p. ISBN: 978-989-9653-7-1

5 - ARRUDA, A. M., ed. (2013) - Fenícios e púnicos, por terra e mar, 1. Actas do VI Congresso Internacional de Estudos Fenícios e Púnicos, Estudos \& memórias 5. Lisboa: UNIARQ. 506 p. ISBN: 978-989-9653-8-8 
6 - ARRUDA, A. M. ed., (2014) - Fenícios e púnicos, por terra e mar, 2. Actas do VI Congresso Internacional de Estudos Fenícios e Púnicos, Estudos \& memórias 6. Lisboa: UNIARQ. 698 p. 978-989-9653-9-5

7 - SOUSA, E. (2014) - A ocupação pré-romana da foz do estuário do Tejo. Estudos \& memórias 7. Lisboa: UNIARQ. 449 p. ISBN: 978-989-99146-0-5

8 - GONÇALVES, V. S.; DINIZ, M.; SOUSA, A. C., eds. (2015) - 5. Congresso do Neolítico Peninsular. Actas. Estudos \& memórias 8. Lisboa: UNIARQ/ FL-UL. 661 p. ISBN: 978-989-99146-1-2

9 - SOUSA, A. C.; CARVALHO, A.; VIEGAS, C., eds. (2016) - Terra e Água. Escolher sementes, invocar a Deusa. Estudos em Homenagem a Victor S. Gonçalves. Estudos \& memórias 9. Lisboa: UNIARQ/ FL-UL. 624 p. ISBN: 978-989-99146-2-9; 978-989-99146-3-6

Clio e todas as edições da UNIARQ em formato A4, à excepção dos primeiros seis volumes dos Cadernos, em formato $19 \times 24,5 \mathrm{~cm}$.

O Sector de publicações da UNIARQ está assim de boa saúde e vários outros títulos estão no prelo e garantem a continuidade, agora sob a coordenação de Ana Catarina Sousa. Como diriam os chineses, que adoram as metáforas floridas, que a Grande Muralha nos proteja as camélias. Ou os japoneses, que são mais concretos, que as cerejeiras continuem a florir e o Gyokuro nunca nos falte (o Gyokuro, o sushi e o sashimi e, já agora, os pastéis de bacalhau e o cozido à portuguesa...).

Victor S. Gonçalves - Verão de 2017 


\section{OPHIUSSA}

\section{POLÍTICA EDITORIAL}

A Ophiussa - Revista do Centro de Arqueologia da Universidade de Lisboa foi iniciada sob a direcção de Victor S. Gonçalves em 1996, tendo sido editado o volume 0. O volume 1 (2017) é uma edição impressa e digital da UNIARQ - Centro de Arqueologia da Universidade de Lisboa.

O principal objectivo desta revista é a publicação e divulgação de trabalhos com manifesto interesse, qualidade e rigor científico sobre temas de Pré-História e Arqueologia, sobretudo do território europeu e da bacia do Mediterrâneo.

A Ophiussa - Revista do Centro de Arqueologia da Universidade de Lisboa publicará um volume anual. A partir de 2018, os artigos submetidos serão sujeitos a um processo de avaliação por parte de revisores externos (peer review). O período de submissão de trabalhos decorrerá sempre no primeiro trimestre e a edição ocorrerá no último trimestre de cada ano.

A revista divide-se em duas secções: artigos científicos e recensões bibliográficas. Excepcionalmente poderão ser aceites textos de carácter introdutório, no âmbito de homenagens ou divulgações específicas, que não serão submetidos à avaliação por pares. Isentas desta avaliação estão também as recensões bibliográficas.

Todas as submissões serão avaliadas, em primeira instância, pela Coordenação Editorial, no que respeita ao seu conteúdo formal e à sua adequação face à política editorial e às normas de edição da revista. Os trabalhos que cumprirem estes requisitos serão posteriormente submetidos a um processo de avaliação por pares cega / blind peer review (mínimo de dois revisores). O Conselho Científico, constituído pela direcção da UNIARQ e por investigadores externos, seleccionará os revisores e acompanhará o processo de edição.

Esta etapa será concretizada por investigadores externos qualificados, sendo os respectivos pareceres entregues num período não superior a três meses. Os revisores procederão à avaliação de forma objectiva, tendo em vista a qualidade do conteúdo da revista; as suas críticas, sugestões e comentários serão, na medida do possível, construtivos, respeitando as capacidades intelectuais do(s) autor(es). Após a recepção dos pareceres, o(s) autor(es) tem um prazo máximo de um mês para proceder às alterações oportunas e reenviar o trabalho.

A aceitação ou recusa de artigos terá como únicos factores de ponderação a sua originalidade e qualidade científica. $\mathrm{O}$ processo de revisão é confidencial, estando assegurado o anonimato dos avaliadores e dos autores dos trabalhos, neste último caso até à data da sua publicação.

Os trabalhos só serão aceites para publicação a partir do momento em que se conclua o processo da revisão por pares. Os textos que não forem aceites serão devolvidos aos seus autores.

O conteúdo dos trabalhos é da inteira responsabilidade do(s) autor(es) e não expressa a posição ou opinião do Conselho Científico ou da Coordenação Editorial.

As ilustrações que não sejam do(s) autor(es) devem indicar a sua procedência. O Conselho Científico e a Coordenação Editorial assumem que os autores solicitaram e receberam autorização para a reprodução dessas ilustrações, e, como tal, rejeitam a responsabilidade do uso não autorizado das ilustrações e das consequências legais por infracção de direitos de propriedade intelectual.

A publicação de textos na Ophiussa - Revista do Centro de Arqueologia da Universidade de Lisboa não implica o pagamento de qualquer taxa nem dá direito a qualquer remuneração económica.

Os textos propostos para publicação devem ser inéditos e não deverão ter sido submetidos a qualquer outra revista ou edição electrónica. Aceitam-se trabalhos redigidos em português, inglês, espanhol, italiano e francês.

Esta edição disponibiliza de imediato e gratuitamente a totalidade dos seus conteúdos, em acesso aberto, de forma a promover, globalmente, a circulação e intercâmbio dos resultados da investigação científica e do conhecimento.

Esta publicação dispõe de uma versão impressa, a preto e branco, com uma tiragem limitada, que será distribuída gratuitamente pelas bibliotecas e instituições mais relevantes internacionalmente, e intercambiada com publicações periódicas da mesma especialidade, que serão integradas na Biblioteca da Faculdade de Letras da Universidade de Lisboa. Conta, paralelamente, com uma versão digital, a cores, disponibilizada no endereço www.ophiussa.letras.ulisboa.pt, onde se pode consultar a totalidade da edição.

Para mais informações contactar: uniarq@letras.ulisboa.pt 


\section{OPHIUSSA}

\section{EDITORIAL POLICY}

Ophiussa - Revista do Centro de Arqueologia da Universidade de Lisboa started under the direction of Victor S. Gonçalves in 1996, with the edition of volume 0. Volume 1 (2017) is a printed and digital edition of UNIARQ - Centro de Arqueologia da Universidade de Lisboa.

The main objective of this journal is the publication and dissemination of papers of interest, quality and scientific rigor concerning Prehistory and Archeology, mostly from Europe and the Mediterranean basin.

Ophiussa - Revista do Centro de Arqueologia da Universidade de Lisboa will publish an annual volume. From 2018, submitted articles will be subject to a peer-review evaluation process. The submission period will always occur in the first quarter of each year and the edition will occur in the last quarter.

The journal is divided into two sections: scientific articles and bibliographic reviews. Exceptionally, texts of an introductory nature may be accepted, in the context of specific tributes or divulgations, which will not be submitted to peerreview evaluation. Exemptions from this evaluation are also the bibliographic reviews.

All submissions will be considered, in the first instance, by the Editorial Board, regarding its formal content and adequacy in face of the editorial policy and the journal's editing standards. Papers that meet these requirements will subsequently be submitted to a blind peer-review process (minimum of two reviewers). The Scientific Council, constituted by the directors of UNIARQ and external researchers, will select the peer-reviewers and follow the editing process.

This stage will be carried out by qualified external researchers, and their feedback will be delivered within a period of no more than two months. The reviewers will carry out the evaluation in an objective manner, in view of the quality and content of the journal; their criticisms, suggestions and comments will be, as far as possible, constructive, respecting the intellectual abilities of the author (s). After receiving the feedback, the author(s) has a maximum period of one month to make the necessary changes and resubmit the work.

Acceptance or refusal of articles will have as sole factors of consideration their originality and scientific quality. The review process is confidential, with the anonymity of the evaluators and authors of the works being ensured, in the latter case up to the date of its publication.

Papers will only be accepted for publication as soon as the peer review process is completed. Texts that are not accepted will be returned to their authors.

The content of the works is entirely the responsibility of the author(s) and does not express the position or opinion of the Scientific Council or Editorial Board.

Illustrations that are not from the author(s) must indicate their origin. The Scientific Council and Editorial Board assume that the authors have requested and received permission to reproduce these illustrations and, as such, reject the responsibility for the unauthorized use of the illustrations and legal consequences for infringement of intellectual property rights.

The publication of texts in Ophiussa - Revista do Centro de Arqueologia da Universidade de Lisboa does not imply the payment of any fee nor does it entitle to any economic remuneration.

Texts proposed for publication must be unpublished and should not have been submitted to any other journal or electronic edition. Works written in Portuguese, English, Spanish, Italian and French are accepted.

This edition immediately and freely provides all of its content, in open access, in order to promote global circulation and exchange of scientific research and knowledge.

This publication has a limited printed edition in black and white, which will be distributed free of charge by the most relevant international libraries and institutions, and exchanged with periodicals of the same specialty, which will be integrated in the Library of Faculdade de Letras of Universidade de Lisboa. It also has a digital version, in color, available at address www. ophiussa.letras.ulisboa.pt, where one can consult the entire edition.

For more information contact: uniarq@letras.ulisboa.pt 


\section{OPHIUSSA}

REVISTA DO CENTRO DE ARQUEOLOGIA DA UNIVERSIDADE DE LISBOA

\section{1 - 2017}

\section{ÍNDICE}

VICTOR S. GONÇALVES - Ophiussa regressa, em digital e, logo de seguida, em papel .... MARCO ANTÓNIO ANDRADE - O sítio pré-histórico do Sobral do Martim Afonso (Sätuatterra de Magos, Portugal): um curioso contexto do Neolítico Final / Calcolítico na marégentesquerda do Baixo Tejo

ANA CATARINA SOUSA - JORGE LOPES - O sítio do Moinho do Custódio (tarruda dos vinthost.

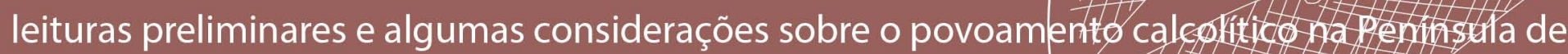
Lisboa

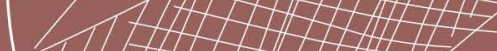
51

PEDRO ALBUQUERQUE - O Guadiana como fronteira? Notas para un profecto-de investigação

ANA MARGARIDA ARRUDA - ELISA DE SOUSA - JOÃO PIMENTA - RUI SOARES - HENRIQU MENDES - Fenícios e indígenas em contacto no Estuário do Tejo

ELISA DE SOUSA - Algumas reflexões sobre a fase tardia da Idade do Ferro no Ocidente Atlântico

CARLOS PEREIRA - Produção e comércio de lucernas durante a Antiguidade Tardia: génese e evolução das lucernas tardo-antigas de produção africana

JACINTA BUGALHÃO - O papel da mulher na Arqueologia Portuguesa

RUI BOAVENTURA - VERA.LEISNER@PORTUGAL.PT

RECENSÕES BIBLIOGRÁFICAS - Estudos Arqueológicos de Oeiras, 22 volumes e a contar... (VICTOR S. GONÇALVES) 